\title{
NUEVOS DATOS PARA EL CONOCIMIENTO DE LAS DIETAS PREHISPÁNICAS DEL DELTA SUPERIOR
}

\author{
Daniel Loponte(1) y Livia Kozameh( $^{(2)}$ \\ (1)Instituto Nacional de Antropología y Pensamiento Latinoamericano y CONICET. \\ 3 de Febrero 1378, (C1426BJN) Ciudad de Buenos Aires. dloponte@inapl.gov.ar \\ ${ }^{(2)}$ Carrera de Investigador Científico, Universidad Nacional de Rosario. \\ Entre Ríos 758, (2000), Rosario. liviak@arnet.com.ar
}

\section{Introducción}

El sitio Cerro Grande de la isla Los Marinos se encuentra en el Departamento de Victoria, en la provincia de Entre Ríos, frente a la ciudad de Rosario, detrás de la cadena de islas (La invernada, Del Espinillo, Castellanos, etc.) que aquí forman la margen izquierda del río Paraná. El sitio fue excavado a mitad del siglo pasado, donde se recuperó una importante cantidad de restos humanos (Gaspary 1950, Kozameh 1993). Los materiales recuperados incluyen una abundante cantidad de cerámica lisa e incisa, incluyendo modelados zoomorfos, y una variada fauna vinculada con el ambiente fluvio-lacustre, característica del área durante el Holoceno tardío. En función de estos datos, y de la información relacionada con la estratigrafía del sitio (Gaspary 1950), se puede considerar que el depósito fue generado por grupos cazadores-recolectores durante el tramo final del Holoceno.

Los estudios de las dietas prehispánicas basadas en los análisis de isótopos estables tienen cierto desarrollo en el extremo meridional del humedal del Paraná inferior, pero ciertamente se desconocen datos referentes al Delta Superior, donde se encuentra el sitio Cerro Grande (ver un resumen en Loponte 2008). Por ello se ha iniciado el estudio de la colección Gaspary, con el fin de extender la base de datos isotópicos que se dispone para presas y humanos del humedal del Paraná inferior. Este trabajo precisamente, constituye el primer avance al respecto.

\section{Materiales y Métodos}

Una fracción de los restos humanos exhumados por Gaspary fueron depositados en 1956 en el Instituto de Antropología de la Facultad de Filosofía y Letras de la Universidad Nacional del Litoral (Rosario) y actualmente se encuentran en el Departamento de Bioantropología y Evolución, Facultad de Humanidades y Artes, Universidad Nacional de Rosario. Los cráneos, que se encuentran en general en muy buen estado de conservación, son los únicos materiales que ingresaron con sus rótulos respectivos. Para los análisis isotópicos se tomaron muestras de 4 individuos adultos ( 2 femeninos y 2 masculinos) consistentes en una pieza dentaria de cada uno. Los estudios fueron realizados en el Center for Applied Isotopes Studies (CAIS UGAMS) de la Universidad de Georgia (USA). Se efectuaron análisis de $\delta^{15} \mathrm{~N}$ y $\delta^{13} \mathrm{C}$, en este último caso se analizó tanto la fracción del colágeno como la fracción inorgánica de las piezas dentales. Además se evaluó el grado de diagénesis de las muestras mediante la razón $\mathrm{C} / \mathrm{N}$.

Por razones de espacio mencionaremos brevemente aquí que los valores de $\delta^{13} \mathrm{C}$ sobre huesos y dientes tienen una directa relación con la absorción de $\mathrm{CO}_{2}$ por parte de los vegetales, 
los cuales pueden adoptar tres vías fotosintéticas diferentes: $\mathrm{C}_{3^{\prime}} \mathrm{C}_{4}$ y CAM. Cada uno de estos grupos funcionales posee una proporción de ${ }^{12} \mathrm{C} /{ }^{13} \mathrm{C}$ relacionada con la evolución y adaptación de los diferentes vegetales (cf. Ambrose 1993; Schoeninger 1995). Las plantas ubicadas en el primer grupo funcional arrojan valores que oscilan entre - 19 y - $35 \%$ o (media $26.5 \%$ ) y las segundas entre -9 y $-15 \%$ o (-12.5\% ) (Ambrose 1993, Van der Merwe et al. 2000). La composición isotópica del tejido humano posee valores enriquecidos en aproximadamente $5 \%$ para el colágeno y $9 \%$ para la apatita debido al consumo de los mismos (Schoeninger 1995). Estudios experimentales demostraron que el colágeno representa la porción proteica de la dieta, mientras que la apatita tiene una relación más cercana con la dieta total (carbohidratos, lípidos y proteínas; Ambrose 1993). Por su parte, el espaciamiento entre ambas fuentes de carbono es menor en los carnívoros que en los herbívoros (Lee-Thorp et al. 1989), lo cual hace posible medir el grado relativo de consumo de carne de un individuo dentro de un contexto ambiental determinado (Van Klinken et al. 2000).

Los valores de $\delta^{15} \mathrm{~N}$ de las plantas oscilan entre $2 \%$ y $6 \%$ (Pate 1994), determinados por su historia evolutiva y el contexto ambiental. El enriquecimiento a medida que asciende a través de la cadena trófica es del orden de 3 - $4 \%$, siendo los valores promedio en mamíferos terrestres 5,7\% medido sobre colágeno óseo (Ambrose 1993; Pate 1994). Dado que el nitrógeno se incorpora en los humanos principalmente como parte de las proteínas, no hay dudas de que a medida que se incrementa su proporción, es mayor la ingesta de proteínas de alto valor trófico (carne y leche). Estos datos generales, sumados a los obtenidos en el extremo meridional del Paraná, donde se ha comenzado a reconstruir la cadena trófica del Holoceno tardío (Loponte 2008; Madanes et al. 2009), permiten integrar e interpretar los datos obtenidos en la muestra proveniente de Cerro Grande.

\section{Resultados}

Los valores obtenidos en las cuatro muestras analizadas indican que los individuos ingirieron plantas y tejidos de animales consumidores de plantas del grupo funcional $\mathrm{C}_{3}$ principalmente, ya que el promedio observado en el colágeno arroja un valor de $\delta^{13} \mathrm{C}-19,57 \%$ o (ver tabla 1). Los valores de la apatita también muestran un neto predominio de grupos funcionales $\mathrm{C}_{3}$, ya que el promedio es -13,43\%. Ambas lecturas están alejadas de aquellas relacionadas con la ingesta de maíz obtenidas en esqueletos incluidos en urnas guaraníes, provenientes de sitios definidos como guaraníes y donde se presume el consumo de esta gramínea (cf. Loponte y Acosta 2008). También son muy diferentes de otras lecturas vinculadas a la ingesta de este cereal dadas a conocer para otras regiones (cf. Van der Merwe et al. 2000; Olivera y Yacobaccio 1999). Por el contrario, son semejantes a aquellas obtenidas en muestras óseas pertenecientes a grupos cazadores-recolectores del Holoceno tardío del extremo meridional del humedal del Paraná inferior. Asimismo, los valores del espaciamiento apatitacolágeno indican una dieta basada en proteínas $\left(\delta^{13} \mathrm{C} 5,96 \%\right.$ ), al menos si la comparamos con tres herbívoros puros del área (ciervo de los pantanos, venado de las pampas y coipo) cuyo valor promedio es $\delta^{13} \mathrm{C} 10,1 \%$, y de otros humanos del tramo final del Paraná inferior $\left(\delta^{13} \mathrm{C} 7,5 \%\right.$ ), ubicados en el bloque cronológico 1100 - 680 años ${ }^{14} \mathrm{C}$ AP (Loponte 2008)..

El importante componente de ingesta de proteínas animales también se corrobora por los altos valores de $\delta^{15} \mathrm{~N}$, cuyo promedio en las cuatro muestras analizadas arroja un valor de $12,62 \%$, alejado del promedio de los herbívoros locales $(\sim 5 \%)$ y de otros humanos con 


\begin{tabular}{|c|c|c|c|c|c|c|c|}
\hline Muestra \# & UGAMS & Género & $\mathrm{C} / \mathrm{N}$ & $\bar{\delta}^{13} \mathrm{C}_{\mathrm{ap} .}(\% \circ)$ & $\overline{0}^{13} \mathrm{C}(\%$ o $)$ & $\Delta^{13} \mathrm{C}$ & $\mathrm{o}^{15} \mathrm{~N}(\% \mathrm{)})$ \\
\hline CG-ILM-7 & 3303 & Masculino & 2,9 & $-14,29 \pm 0,10$ & $-19,16 \pm 0.01$ & 4,87 & $12,66 \pm 0,30$ \\
\hline CG-ILM -21 & 3304 & Masculino & 2,9 & $-14,59 \pm 0,10$ & $-19,86 \pm 0,06$ & 5,27 & $12,31 \pm 0,17$ \\
\hline CG-ILM-26 & 3305 & Femenino & 2,9 & $-12,98 \pm 0,10$ & $-20,73 \pm 0,23$ & 7,02 & $12,73 \pm 0,13$ \\
\hline CG-ILM-123 & 3306 & Femenino & 2,9 & $-11,88 \pm 0,10$ & $-18,56 \pm 0,07$ & 6,68 & $12.79 \pm 0,22$ \\
\hline
\end{tabular}

Tabla 1. Valores isotópicos de 4 individuos procedentes del sitio arqueológico Cerro Grande de la Isla de los Marinos. Delta Superior del Paraná.

mayor consumo de vegetales $(\sim 8,9 \%$ ) recuperados en el tramo final del Paraná (Loponte 2008).

Si bien la muestra es aún pequeña, debe señalarse un incipiente agrupamiento de los resultados según el género de los individuos. En efecto, los dos valores isotópicos relevados muestran un mayor espaciamiento entre ambas fuentes de carbono de los individuos femeninos y un valor más pesado en el carbono de la fracción orgánica (tabla 1). En este sentido, es notable que la cantidad de caries observadas en las mujeres es muy superior a la de los hombres, mostrando una clara tendencia al respecto dentro de la colección (Kozameh ms.). La ampliación de las lecturas permitirá corroborar si existen tendencias divergentes en los datos isotópicos según el género. Asimismo, los resultados obtenidos en la muestra proveniente de Cerro Grande serán mejor contextualizados una vez que logremos ampliar los datos sobre la cadena trófica del Delta Superior del Holoceno tardío, incorporando a esta discusión los fechados radiocarbónicos asociados.

\section{Bibliografía}

Ambrose, S. H.

1993. Isotopic analysis of paleodiets: Methodological and interpretive considerations. Investigations of ancient human tissue. Chemical analysis in anthropology. Editado por M. K. Sandford, pp. 59-130. Pensylvania, Gordon and Breach Science Publishers.

Gaspary, F.

1950. Investigaciones Arqueológicas y Antropológicas en un "cerrito" de la isla Los Marinos (prov. de Entre Ríos). Publicaciones del Instituto de Arqueología, Lingüística y Folklore "Dr. Pablo Cabrera". Universidad Nacional de Córdoba.

Kozameh, L.

1993.Patrones de abrasión dentaria en dos poblaciones prehistóricas argentinas. Boletín de la Sociedad Española de Antropología Biológica 14: 81 - 104. Madrid.

Kozameh, L.

Patologías dentales en poblaciones de cazadoras-recolectores y horticultoras del tramo final de la cuenca del Plata. Ms.

Lee-Thorp, J. A., J. C. Sealy y N. J. Van der Merwe.

1989. Stable isotope carbon ratio differences between bone collagen and and bone apatite, and their relationship to diet. Journal of Archaeological Science 16: 585-599. 
Loponte D.

2008. Arqueología del humedal del Paraná inferior (Bajíos Ribereños meridionales). Serie Monográfica "Arqueología de la Cuenca del Plata", editado por Alejandro Acosta y Daniel Loponte. Instituto Nacional de Antropología y Pensamiento Latinoamericano. Buenos Aires.

Loponte, D. y A. Acosta.

2008. Estado actual y perspectivas de la arqueología de la "Tradición Tupiguaraní" en Argentina. Arqueología Guaraní do Brasil, Os Ceramistas da TradiçãoTupigruaraníl. Editado por T. Andrade Lima y A. Prous, pp.: 197-215. Río de Janeiro.

Madanes, N., R., D. Quintana, M. Biondini y D. Loponte.

2009. Relationships between photosynthetic types in the composition of herbivore diets and environment in the Rio de la Plata Basin, Argentina. Revista Chilena de Historia Natural. En prensa.

Olivera, D. E. y H. D. Yacobaccio .

1999. Estudios de paleodieta en poblaciones humanas de los Andes del Sur a través de isótopos estables. Trabajo presentado al V Congreso Nacional de Paleopatología, Alcalá La Real, Jaén.

Pate, F. D.

1994. Bone Chemistry and Paleodiet. Journal of Archaeological Method and Theory 1:161-209.

Schoeninger, M. J.

1995. Stable Isotopes Studies in Human Evolution. Evolutionary Anthropology 4 (3): 83- 98.

Van der Merwe, N. J., R. H. Tykot, N. Hammond y K. Oakberg.

2000. Diet and animal husbandry of the Preclassic Maya at Cuello, Belize: isotopic and zooarchaeological evidence. En Bioarchaeological approaches to paleodietary analysis. Editado por S. H. Ambrose y N. A. katzemberg, pp. 23-38.

Van Klinken, G.J., M. P. Richards y R. E. M. Hedges

2000. An overview of causes for stable isotopic variations in past European human population: environmental, ecophysiological and cultural effects. En Bioarchaeological approaches to paleodietary analysis. Editado por S. H. Ambrose y N. A. katzemberg, pp. 39-63. 